\title{
Cambios en la arquitectura muscular y en la velocidad de ejecución de sentadillas en VersaPulley en condiciones estables e inestables en jugadores junior de baloncesto de élite durante una temporada
}

\author{
Changes in muscular architecture and execution velocity \\ during squats performed using the VersaPulley under stable \\ and unstable conditions in junior elite basketball players
}

\section{Mudanças na arquitetura muscular e agachamentos velocidade de execução em VersaPulley em condiçôes estáveis e instáveis em juniores jogadores de basquete de elite durante a temporada}

\author{
Jairo Vázquez-Guerrero ${ }^{1,2}$ y Gerard Moras
}

Resumen: Los objetivos del presente estudio fueron: a) monitorizar y comparar la velocidad de ejecución de sentadillas con VersaPulley (VP) en condiciones estables (CE) y condiciones inestables (CI) en jugadores junior de baloncesto de élite durante una temporada y b) evaluar los cambios en la arquitectura muscular (AM). Siete jugadores junior de baloncesto realizaron el entrenamiento técnico-táctico y de acondicionamiento físico de la temporada (16 horas semanales) incluyendo entrenamiento de fuerza mediante sentadillas con VP en CE y CI. Durante la temporada se evaluó la velocidad $_{\text {pico }}$ de ejecución de las sentadillas mediante el sistema T-FORCE. Además, cada 3 meses se valoró la AM del vasto lateral mediante ultrasonidos. Los resultados no mostraron diferencias significativas en la velocidad $_{\text {pico }}$ al realizar sentadillas entre ambas condiciones en ningún momento de la temporada, aumentando un $17 \%$ en CE y un $23 \%$ en CI ( $<<0,01)$. El grosor muscular $(\mathrm{p}=0,009)$ y el ángulo de peneación $(\mathrm{p}<0,05)$ aumentaron mientras que la longitud de los fascículos no experimentó cambios significativos. El entrenamiento regular de baloncesto y el entrenamiento de fuerza incluyendo sentadillas con VP en CE y CI generaron niveles similares de velocidad $_{\text {pico }}$ en ambas condiciones en jugadores de baloncesto júnior de élite. La velocidad ${ }_{\text {pico }}$ aumentó tanto en CE como en CI durante la temporada. Además, se produjeron cambios en la AM.

Palabras clave: entrenamiento de fuerza, inestabilidad, inercia rotacional, grosor muscular, ángulo de peneación, baloncesto, jóvenes.

Abstract: The purposes of the present study were to: a) monitor and compare velocity ${ }_{\text {peak }}$ when performing squats with VersaPulley (VP) on stable conditions (CE) and on unstable conditions (CI) in elite junior basketball players during a season and $\mathrm{b}$ ) assess the changes in muscle architecture (AM). Seven junior basketball players performed the technical-tactical training and the physical fitness of the season (16 weekly hours) adding extra strength training performing squats with the VP under CE and CI.

Dirección para correspondencia [Correspondence address]: Jairo Vázquez-Guerrero, Instituto Nacional de Educación Física de Barcelona. Avinguda de l'Estadi, 12, 08038 Barcelona (Espańa). E-mail: jairosport. vg@gmail.com
Velocity $_{\text {peak }}$ when performing the squats was assessed during the season through T-FORCE system. Moreover, every 3 months the AM of the vastus lateralis was assessed. Results indicated that velocity $y_{\text {peak }}$ did not show significant differences when performing squats under both conditions during the season. Velocity ${ }_{\text {peak }}$ increased $17 \%$ under $\mathrm{CE}$ and $23 \%$ under CI $(\mathrm{p}<0.01)$. The muscle thickness $(\mathrm{p}=0.009)$ and the pennation angle $(\mathrm{p}<$ 0.05 ) increased whilst the fascicle length did not show significant differences. The regular basketball training and the strength training, including squats performed with VP under CE and CI, increased a long de season in a basketball elite junior players. Moreover, we founded changes in AM. Key words: strength training, instability, rotational inertia, muscular thickness, pennation angle, basketball, youth.

Resumo: Os objetivos do estudo em questão foram: a) monitorar e comparar a velocidade máxima de execução de agachamento no VersaPulley (VP) em condiçôes estáveis (CE) e condiçôes instáveis (CI) em jogadores juvenis de basquete de elite durante uma temporada e b) avaliar as mudanças na arquitetuta muscular (AM). Sete jogadores juvenis de basquete realizaram o treinamento técnico-tatico e de condicionamento físico da temporada (16 horas semanais) incluindo treinamento de forca através de agachamentos com VP em CE e CI. Durante a temporada monitorizou-se a velocidade máxima de execução dos agachamentos através do sistema T-FORCE. Além disso, a cada 3 meses avaliou-se a AM do vasto lateral através de Ultra-som. Os resultados nao mostraram diferenças significativas na velocidade máxima ao realizar agachamentos entre ambas condiçóes em nenhum momento da temporada, aumentando um $17 \%$ em CE e um $23 \%$ em CI $(p<0,01)$ A grossura muscular $(\mathrm{p}=0,009)$ e o ângulo de penaçáo $(\mathrm{p}<0,05)$ aumentaram enquanto que a longitude dos fascículos nao demonstrou mudanças sinificativas. $\mathrm{O}$ treinamento normal de basquete e o treinamento de força incluindo agachamentos com VP em CE e CI geraram níveis similares de velocidade máxima em ambas condiçóes nos jogadores de basquete juvenis de elite. A velocidade maxima aumentou tanto em CE como em CI durante a temporada. Além disso, produziu-se mudanças na AM.

Palavras chave: treinamento de força, instabilidade, espessura do músculo, ângulo pennation, basquete, jovem. 


\section{Introducción}

La alta exigencia del baloncesto produce importantes cargas fisiológicas sobre los jugadores durante la competición (McInnes, Carlson, Jones, y McKenna, 1995). En el baloncesto moderno se producen unas 1000 acciones por partido (Ben Abdelkrim, El Fazaa, y El Ati, 2007) de las cuales el 11,5\% son de intensidad máxima (Ben Abdelkrim y cols., 2010). La duración de las acciones de alta intensidad (Ben Abdelkrim y cols., 2007; Hoffman y Maresch, 2000) oscila entre 2 y 5 s, predominando las de 2 s (Ben Abdelkrim y cols., 2007), con una densidad de 1:10 en las acciones alta intensidad (Ben Abdelkrim y cols., 2010; Hoffman y Maresch, 2000; McInnes y cols., 1995). En consecuencia, se considera el acondicionamiento físico un factor importante para preparar a los jugadores de baloncesto de élite moderno (Apostolidis, Nassis, Bolatoglou, y Geladas, 2004; Sallet, Perrier, Ferret, Vitelli, y Baverel, 2005; Simenz, Dugan, y Ebben, 2005).

Los movimientos realizados por los jugadores son el resultado de la fuerza generada por la acción de múltiples músculos (Oshita y Yano, 2010). Así, ninguna actividad física del ser humano es posible sin la fuerza muscular (Ehlenz, Grosser, y Zimmermann, 1990). Existe suficiente evidencia científica para incluir de forma integral los programas de entrenamiento de fuerza como parte dela preparación en los deportes de equipo (McGuigan, Wright, y Fleck, 2012). Concretamente, la capacidad para generar la máxima fuerza en el menor período de tiempo ha sido considerada en baloncesto, esencial en la obtención de altos niveles de rendimiento deportivo (Hedrick, 1993; Hoffman, Tenenbaum, Maresh, y Kraemer, 1996; Klinzing, 1991).

Existen diferentes metodologías o sistemas de entrenamiento de fuerza y potencia que han aumentado en popularidad en las últimas décadas, entre otros, el entrenamiento de inercia rotacional (Berg y Tesch, 1994; Berg y Tesch, 1998; M. Naczk, A. Naczk, W. Brzenczek-Owczarzak, J. Arlet, y Z. Adach, 2013; Norrbrand, Fluckey, Pozzo, y Tesch, 2008; Norrbrand, Pozzo, y Tesch, 2010) y el entrenamiento en condiciones de inestabilidad (Behm y Colado, 2012; Behm, Anderson, y Curnew, 2002; Behm y Anderson, 2006; Behm, Leonard, Young, Bonsey, y MacKinnon, 2005; Drinkwater, Pritchett, y Behm, 2007; Saeterbakken y Fimland, 2013; Zemková y cols., 2013; Zemková y cols., 2012) que podrían optimizar el rendimiento deportivo. El entrenamiento mediante dispositivos de inercia rotacional utiliza la resistencia de inercia rotacional ofreciendo resistencia independiente de la gravedad y difiere de otras modalidades tradicionales que utilizan principalmente el peso libre. Durante este tipo de entrenamiento se producen breves episodios de sobrecarga excéntrica generando un pico máximo de fuerza mayor que el obtenido habitualmente durante el entrenamiento tradicional (Norrbrand y cols., 2008; Norrbrand y cols., 2010).
Así, varios estudios han confirmado la eficacia de este entrenamiento para mejorar la fuerza y la potencia (Albert, Hillegass, y Spiegel, 1994; Caruso y cols., 2006; Mariusz Naczk, Alicja Naczk, Wioletta Brzenczek-Owczarzak, Jaroslaw Arlet, y Zdzislaw Adach, 2013; Onambélé y cols., 2008; Tesch, Ekberg, Lindquist, y Trieschmann, 2004). Por otro lado, el entrenamiento de fuerza en CI podría aumentar la solicitación sobre el sistema neuromuscular, además de mejorar el equilibrio y la coordinación, siendo más específico del deporte. Esto podría mejorar el rendimiento deportivo. Gran parte de la literatura científica ha investigado las respuestas agudas y crónicas al entrenamiento en CI (fuerza y rendimiento, equilibrio, estabilidad, valoración del rendimiento y electromiografía) tal como reflejan Behm yColado (2012) en una revisión. Así, existe un amplio consenso al afirmar que la fuerza, la potencia y la velocidad disminuyen al realizar ejercicios en CI.

Además de describir los cambios en el rendimiento, es fundamental investigar los mecanismos que el entrenamiento de fuerza y la práctica deportiva producen a nivel muscular. Se ha sugerido previamente que la arquitectura muscular (AM) puede explicar mejor la fuerza máxima y la velocidad que las propiedades químicas intrínsecas (Wickiewicz, Roy, Powell, y Edgerton, 1983) . La AM, que incluye medidas del grosor del músculo, ángulo de peneación y longitud de fascículos mediante ecografía ha demostrado ser fiable (Aagaard y cols., 2001; Blazevich, Gill, Bronks, y Newton, 2003; Blazevich, Gill, Deans, y Zhou, 2007; Seynnes, de Boer, y Narici, 2007) y su capacidad de adaptación en respuesta al entrenamiento de fuerza (Aagaard y cols., 2001; Alegre, Jiménez, GonzaloOrden, Martín-Acero, y Aguado, 2006; Blazevich, Cannavan, Coleman, y Horne, 2007; Blazevich y cols., 2003; Kawakami, Abe, Kuno, y Fukunaga, 1995; Seynnes y cols., 2007). Sin embargo, sólo 3 estudios han investigado los cambios en la AM y el rendimiento deportivo realizando de forma concurrente el entrenamiento de su deporte y de fuerza (Blazevich y cols., 2003; Jajtner y cols., 2013; Nimphius, McGuigan, y Newton, 2012). Ninguno de ellos fue realizado con jugadores junior de élite de baloncesto.

La velocidad, potencia y fuerza de la rodilla y de los extensores de cadera son vitales para mejorar el rendimiento en los deportes. Muchos entrenadores de fuerza proponen la sentadilla como un ejercicio esencial para optimizar el rendimiento deportivo (Comfort, Bullock, y Pearson, 2012; Chandler, 1992; McBride y cols., 2009). Sin embargo, no se conocen estudios que combinen el entrenamiento de sentadilla utilizando un dispositivo VP en CE y CI y su monitorización mediante la velocidad ${ }_{\text {pico }}$ durante una temporada en jugadores de baloncesto de élite.

Por tanto, los objetivos de este estudio fueron a) monitorizar y comparar los cambios en la velocidad ${ }_{\text {pico }}$ al realizar sentadillas con VP en CE y CI en jugadores junior de baloncesto 
de élite durante una temporada y b) evaluar los cambios en la AM. Las hipótesis fueron que a) la velocidad ${ }_{\text {pico }}$ sería mayor en CE que en CI, b) la velocidad ${ }_{\text {pico }}$ aumentaría de forma similar en ambas condiciones durante la temporada y c) se producirían cambios en la AM.

\section{Material y método}

\section{Diseño}

Para evaluar las adaptaciones generadas por el entrenamiento en un equipo junior de baloncesto de élite durante una temporada se llevó a cabo un estudio longitudinal evaluando y comparando los cambios en la velocidad ${ }_{\text {pico }}$ al realizar sentadillas en CE y CI. Se analizaron las adaptaciones producidas en la AM. Después de varias semanas de familiarización con el ejercicio de sentadilla en VP en CE y CI y de preparación general se realizó el ejercicio de sentadilla a la máxima velocidad posible con VP en ambas condiciones de forma aleatoria sirviendo como test para controlar y evaluar la velocidad alcanzada en ese ejercicio. Este ejercicio se repitió y se monitorizó cada 7 ó 15 días durante 22 semanas. Además, se evaluó la AM del vasto lateral cada 3 meses.

\section{Participantes}

Siete jugadores de baloncesto (edad: 16,75 \pm 0,5 ańos; altura: $200,09 \pm 2,55 \mathrm{~cm}$; peso corporal 89,97 $\pm 9,96 \mathrm{~kg}$ ) del mismo club, con experiencia competitiva en la selección nacional en campeonatos mundiales o europeos realizaron el entrenamiento regular de baloncesto de la temporada y el entrenamiento de fuerza (12 y 4 horas semanales respectivamente) incluyendo la implementación de entrenamiento de fuerza con VP. Todos los participantes fueron informados del estudio firmando el correspondiente consentimiento informado. La aprobación de esta investigación fue concedida por el Comité de Ética y Científico y el estudio se ajusta a la Declaración de Helsinki para la investigación médica en seres humanos.

\section{Entrenamiento}

Durante la temporada se realizó el entrenamiento regular de baloncesto que incluía en un microciclo estándar 4-5 horas de entrenamiento para cada una de las estructuras (técnica, táctica y condicional), además del partido oficial disputado durante el fin de semana. En cuanto a la estructura condicional, las 3-4 sesiones semanales realizadas eran destinadas fundamentalmente al entrenamiento de fuerza, puesto que la resistencia y la velocidad estaban incluidas de forma integrada en el entrenamiento de pista. Así, el objetivo principal era el desarrollo de la hipertrofia muscular, además de optimizar la potencia muscular y la prevención de lesiones.

\section{Instrumentos}

$\mathrm{VP}^{\odot}$

El dispositivo VP consta de un volante de inercia rotacional de metal y un eje fijo en el centro sobre el cual giran unas masas. Para variar la carga (inercia), este dispositivo permite colocar un total de 16 masas independientes situadas en el borde del volante. Un cono situado encima del volante hace posible que una cuerda fijada en su extremo superior se enrolle y desenrolle alrededor del mismo. La longitud de la cuerda aumenta cuando se desenrolla (fase concéntrica). Una vez la cuerda se ha desenrollado completamente, el cono sigue girando y la cuerda empieza a enrollarse alrededor de este (fase excéntrica). Para variar el ratio fuerza/velocidad es posible seleccionar 3 posiciones modificando la ubicación de la polea más cercana al cono. En la posición superior de la polea, donde la cuerda gira alrededor de la parte más estrecha del cono se producen los menores niveles de velocidad, mientras que en la posición inferior que utiliza un diámetro más amplio del cono para enrollar la cuerda, se alcanzan las velocidades más altas. En la presente investigación se utilizaron 16 masas en la posición 2 (intermedia). Dadas las características de este dispositivo, ante una misma inercia, una mayor velocidad en la ejecución del ejercicio supone mayores niveles de producción de fuerza.

\section{Pielaster $^{\circ}$}

El Pielaster (Biolaster, S.L., Guipúzcoa, Spain) es una plataforma inestable esferoidal de planta elíptica, que sigue el contorno del pie y permite un giro ondulante de $360^{\circ}$ si está colocado sobre su porción convexa. Esta forma específica del Pielaster provoca que el brazo de palanca sea diferente en función de la posición, consiguiendo adaptar la fuerza necesaria para mantener el equilibrio a la fuerza muscular de los músculos involucrados en ese movimiento específico.

\section{Procedimiento}

Al finalizar el periodo preparatorio y después de una fase de familiarización con el ejercicio de sentadilla con VP en CE y CI en la que se puso el énfasis en la adecuada ejecución técnica de la sentadilla, alcanzando un ángulo de $90^{\circ}$ en la flexión de rodillas, los participantes realizaron un test de 8 repeticiones ejecutando sentadillas a la máxima velocidad en una plataforma estable y encima de dos Pielasters. De este modo al inicio de periodo experimental se determinó la velocidad de ejecución de este ejercicio (test 1) en ambas condiciones.

El test se realizó después de un calentamiento estandarizado. Los jugadores realizaron de forma aleatoria un test de 8 repeticiones a máxima velocidad en cada una de las condiciones. Se proporcionó un intervalo de descanso de 2 minutos 
entre cada una de las series (CE y CI). Los resultados de las 8 repeticiones de cada jugador en ambas condiciones fueron grabados para su posterior evaluación. Durante la temporada y siguiendo el mismo protocolo realizaron un total de 19 test incluidos en las sesiones de acondicionamiento físico como un ejercicio del programa de entrenamiento de fuerza. La ejecución del ejercicio requería que el participante utilizara un chaleco ajustable unido a la cuerda de la VP y al cable del encoder lineal del T-FORCE a través de un mosquetón, de forma que la cuerda y el cable quedaban perpendiculares al suelo. El jugador colocaba los pies delante de la VP a ambos lados de la polea anclada en el suelo, con una separación correspondiente a la anchura de la cadera (Figura 1). Esta distancia fue marcada en el suelo y se mantuvo en ambas condiciones. Finalmente, la tensión de la cuerda se ajustaba mientras se mantenían las piernas extendidas. Entonces, se iniciaba la rotación del volante del dispositivo VP enrollando la cuerda hasta alcanzar los $90^{\circ}$ de flexión de rodillas determinados a partir de inspección visual. En ese momento, el sujeto iniciaba el movimiento de extensión aumentando progresivamente la velocidad hasta casi alcanzar la velocidad máxima en la tercera repetición, realizando entonces 8 repeticiones a la máxima velocidad posible. La sentadilla se realizó encima de losPielasters y sobre una plataforma estable de madera específicamente diseñada para mantener los pies a la misma altura respecto al suelo en las dos condiciones. Cada repetición se llevó a cabo hasta alcanzar unos $90^{\circ}$ de ángulo de la rodilla. Para asegurar el esfuerzo máximo y una técnica apropiada se proporcionó estímulo verbal.

Figura 1. VP, sistema de medición T-FORCE, plataforma estable y participante realizando el ejercicio de sentadila encima del Pielaster.

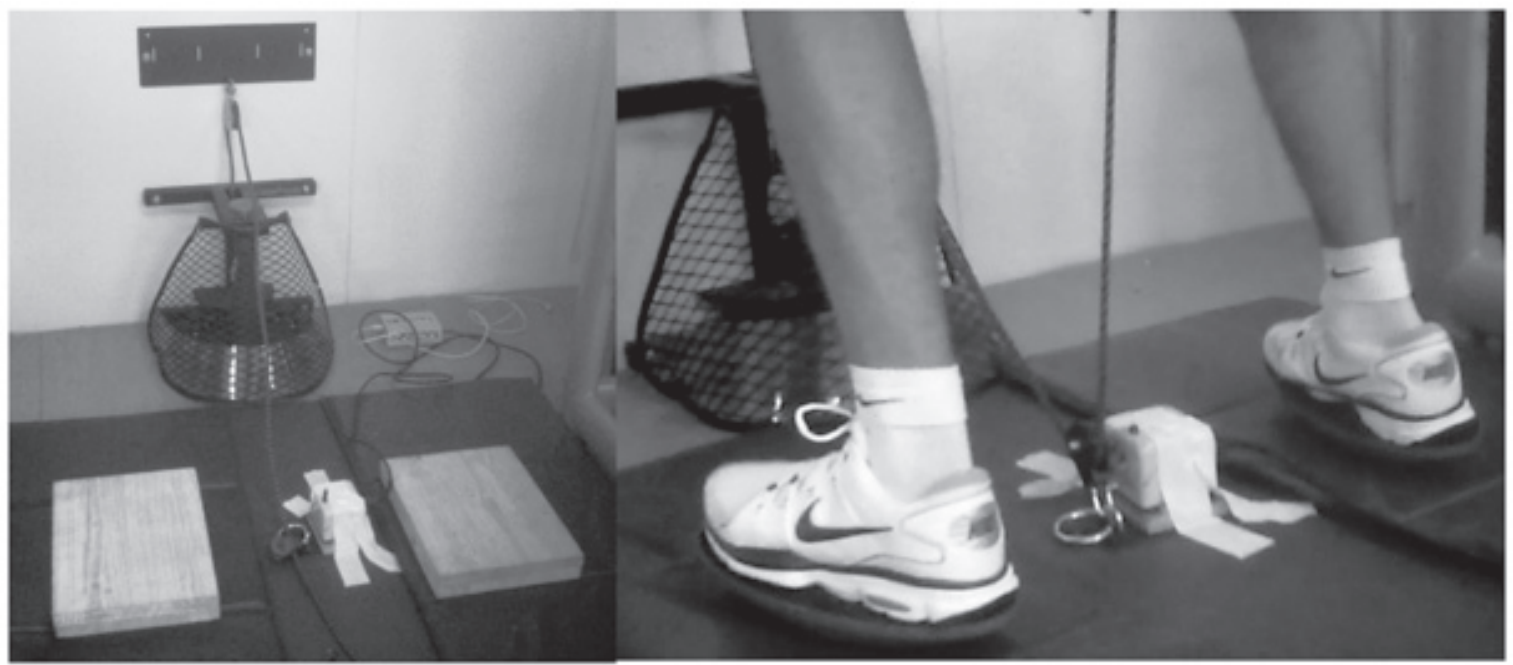

La velocidad del movimiento en sentadilla se midió utilizando un dinamómetro isoinercial (T-Force Dynamic Measurement System Ergotech, Murcia, España) que recogía el desplazamiento vertical del sujeto. Este sistema consta de un transductor de velocidad lineal con un cable interconectado a un ordenador personal con una resolución de adquisición de datos AD de 14 bits y software propio. La velocidad vertical instantánea era muestreada directamente por el dispositivo a una frecuencia de $1000 \mathrm{~Hz}$. El encoder lineal se colocó entre los pies justo al lado de la polea anclada en el suelo. Para medir la aceleración total $\left(3.94 \pm 1.27 \mathrm{~m} \cdot \mathrm{s}^{-2}\right)$ y estimar de forma indirecta la inestabilidad generada se utilizó un acelerómetro de tres ejes $10 \mathrm{~g}$ (Mega Electronics Ltd., Finlandia) fijado con cinta adhesiva en el borde superior del Pielaster derecho y conectado a un convertidor AD de 14 bits (ME6000 Biomonitor, Mega Electrónica, Kuopio, Finlandia). Las señales fueron muestreadas a una frecuencia de $2000 \mathrm{~Hz}$. El acelerómetro se calibró sobre dos puntos de calibración mediante la apli- cación de gravedad cero y gravedad de la Tierra de $1 \mathrm{~g}(9,81$ $\left.\mathrm{m} \cdot \mathrm{s}^{-2}\right)$. Se calculó el vector de la aceleración total mediante la combinación cuadrática de los valores de los ejes X, Y y Z.

AM

La medición de ultrasonidos se realizó en base a protocolos descritos anteriormente (Aagaard y cols., 2001; Abe, Brechue, Fujita, y Brown, 1998; Blazevich, Cannavan, y cols., 2007; Blazevich y cols., 2003; Blazevich, Gill, y cols., 2007; Kawakami, Abe, y Fukunaga, 1993; Kubo y cols., 2003). Todas las imágenes de ultrasonidos fueron grabadas con un ecógrafo LOGIQ e (General Electric Healthcare, Milwaukee, Wisconsin) en modo B, con un cabezal lineal a $10 \mathrm{MHz}$. Las imágenes fueron analizadas con el software R6.x.x. Se tomaron imágenes sagitales del vasto lateral, al 50\% de la distancia desde el trocánter mayor del fémur hasta el epicóndilo lateral del fémur (Alegre y cols., 2006; Blazevich, Cannavan, y cols., 2007; Reeves, Narici, 
y Maganaris, 2004; Rutherford y Jones, 1992; Seynnes y cols., 2007). Se tomaron 3 imágenes de la pierna dominante (Kearns, Isokawa, y Abe, 2001). Todas las mediciones fueron realizadas por el mismo examinador asegurándose de que la musculatura analizada estuviera relajada. Se aplicó gel soluble en agua al transductor antes de ser aplicado a la piel, lo que permitía el acoplamiento adecuado sin un exceso de presión que pudiera causar deformación y alterar las medidas. El transductor se colocó perpendicular a la piel con una orientación paralela a los fascículos basada en la disposición de los fascículos de cada uno de los sujetos hasta que la imagen era considerada adecuada. El grosor muscular fue medido como la distancia perpendicular entre la aponeurosis profunda y superficial del músculo. El ángulo de peneación fue medido como el ángulo entre la dirección de los fascículos y la aponeurosis profunda del músculo. La longitud de fascículos se calculó mediante trigonometría a partir del grosor muscular y del ángulo de peneación según la ecuación: [Longitud de fascículos = Grosor muscular / Seno $\alpha$. La reproducibilidad de los procedimientos descritos para la AM ha demostrado ser altamente fiable y válida (Blazevich y Giorgi, 2001; Kawakami y cols., 1993). El coeficiente de correlación intraclase y el coeficiente de variación de las medidas examinadas fueron determinados antes de la recogida de datos siendo 0,91 y $2,5 \%$ para el grosor, 0,84 y $5,1 \%$ para el ángulo y 0,72 y $6,2 \%$ para la longitud de los fascículos respectivamente.

\section{Análisis estadístico}

Los datos fueron analizados utilizando el programa estadístico PASW Statistics 18 (antes SPSS Statistics) (SPSS, Inc., Chicago, IL, EE.UU.). Las diferencias estadísticamente significativas se fijaron en $\mathrm{p}<0,05$. Los supuestos del modelo fueron validados mediante la prueba de Kolmogorov-Smirnov de normalidad y prueba de la igualdad de las varianzas de Levene. Se analizaron la velocidad ${ }_{\text {pico }}$ y el recorrido utilizando un ANOVA de 2 vías con medidas repetidas considerando la condición (estable e inestable) y los test (1 al 19). Cuando se encontró un efecto estadísticamente significativo se realizaron comparaciones post-hoc. Se utilizó el método de corrección de Bonferroni para comparaciones múltiples.
Las variables de arquitectura muscular fueron analizadas mediante la prueba no paramétrica de Friedman.

\section{Resultados}

La figura 2 muestra los cambios en la velocidad ${ }_{\text {pico }}$ al realizar sentadillas en CE y CI. La velocidad ${ }_{\text {pico }}$ aumentó un 17\% en CE y un $23 \%$ en CI entre la primera y la última valoración (efecto del tamaño=1,78 y 1,71 respectivamente), mostrando diferencias significativas entre la primera y cada una de las restantes sesiones $(p<0,01)$. Sin embargo, no se observaron diferencias significativas entre ambas condiciones en ninguna de las sesiones $(p<0,481)$.

No se hallaron diferencias significativas en el recorrido $(p<0,458)$ entre ambas condiciones.

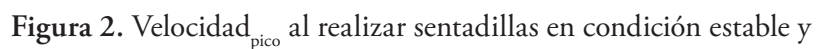
condición inestable durante la temporada regular. ${ }^{*} \mathrm{p}<0,01$ diferencias significativas entre el test 1 y cada uno de los test realizados $n=7$.

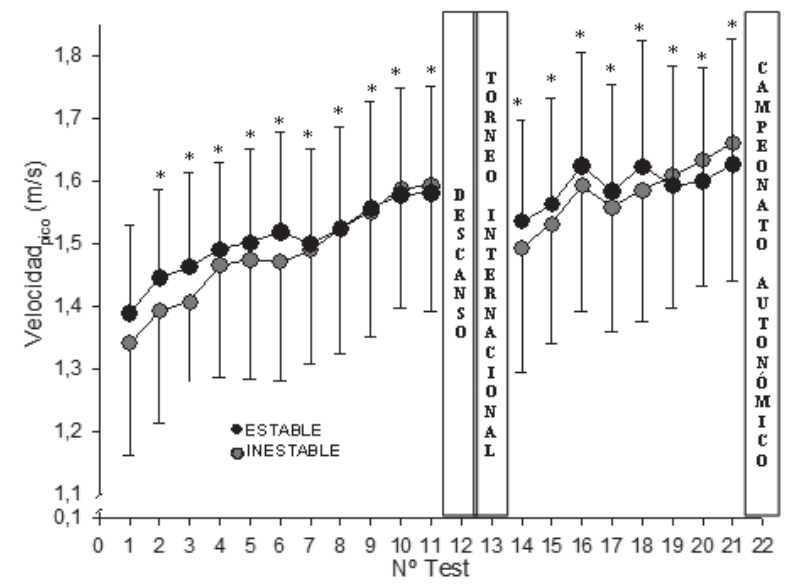

La tabla 1 presenta los resultados de las evaluaciones pre, post1 y post- 2 de la AM. Los resultados pre y post- 2 muestran un aumento del grosor muscular y del ángulo de peneación ( $8,8 \%, p=0,008$ y $8,7 \%, p<0,05$ respectivamente) pero no de la longitud de los fascículos $(\mathrm{p}=1)$.

Tabla 1. Cambios en arquitectura muscular durante la temporada.

\begin{tabular}{lcccc}
\hline & Pre & Post-1 & Post- & Tamańo del efecto Pre-Post-2 \\
\hline Altura $(\mathrm{cm})$ & $200,09 \pm 2,55$ & $200,43 \pm 2,77$ & $201,57 \pm 3,28$ & 0,58 \\
Peso $(\mathrm{kg})$ & $89,97 \pm 9,96$ & $91,52 \pm 9,44$ & $94,21 \pm 7,32^{* \dagger}$ & 0,43 \\
Grosor VL $(\mathrm{cm})$ & $2,51 \pm 0,27$ & $2,67 \pm 0,26^{* *}$ & $2,75 \pm 0,28^{* *}+\dagger$ & 0,89 \\
Ángulo VL $\left(^{0}\right)$ & $18,34 \pm 1,89$ & $19,54 \pm 1,06^{*}$ & $20,11 \pm 1,27^{*} \dagger$ & 0,94 \\
LF VL $(\mathrm{cm})$ & $8,00 \pm 0,67$ & $8,01 \pm 0,78$ & $8,01 \pm 0,75$ & 0,01 \\
\hline
\end{tabular}

VL: vasto lateral, LF: longitud fascículo

${ }^{* *} p<0,009,{ }^{*} p<0,05$; diferencias entre Pre,Post-1 y Pre-Post-2.

$\dagger \uparrow p<0,009, \uparrow p<0,05$ : diferencias entre Post-1 y Post-2 


\section{Discusión}

Los hallazgos claves de este estudio incluyen: a) la capacidad de jugadores junior de baloncesto de alto nivel de generar niveles similares de velocidad ${ }_{\text {pico }}, y$ por tanto de fuerza, al realizar sentadillas con VP en CE y CI, b) la capacidad de aumen-

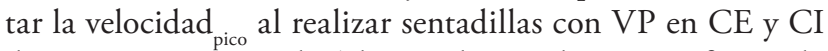
durante una temporada c) los cambios en la AM. La fuerza de esta investigación incluye fundamentalmente ser un estudio longitudinal que permite examinar los cambios producidos en los diferentes parámetros analizados en jóvenes jugadores de baloncesto de alto nivel. Sin embargo, una limitación es el reducido tamaño de la muestra lo que requiere una interpretación cautelosa de los resultados.

En desacuerdo con la primera hipótesis, se halló una gran similitud en la velocidad ${ }_{\text {pico }}$ en ambas condiciones, siendo un 3\% inferior en CI en el promedio de la temporada. Diferentes autores han demostrado una reducción en la fuerza, la potencia y la velocidad cuando aumenta la inestabilidad. La inconsistencia entre las investigaciones previas y este estudio podrían ser atribuidas al grado de inestabilidad durante la tarea (disco de equilibrio inflable, Bosu ball, bloques foam vs Pielaster), al tipo de acción muscular (isométrico vs anisométrico) y al equipamiento (peso libre y barra olímpica vs VP). Drinkwater y cols. (2007) utilizaron bloques foam y bosu ball al realizar sentadillas con diferentes cargas (barra olímpica de $20 \mathrm{~kg}$, 40\% 10RM y $100 \% 10$ RM) hallando reducciones en las medidas de fuerza concéntrica $_{\text {pico }}$ potencia ${ }_{\text {pico }} \mathrm{y}_{\text {velocidad }}$ pico. Concretamente, la velocidad ${ }_{\text {pico }}$ se redujo $0,15,0,15$ y $0,22 \mathrm{~m} / \mathrm{s}$ al realizar el ejercicio encima del bosu ball y 0,02, 0,04 y $0,03 \mathrm{~m} / \mathrm{s}$ en $\mathrm{los}$ bloques foam, mientras que en nuestro estudio fue de 0,016 $\mathrm{m} / \mathrm{s}$ en el promedio de todas las sesiones. De esta forma, la disminución de la velocidad ${ }_{\text {pico }}$ al realizar sentadillas con VP encima de los Pielasters fue claramente menor en comparación con el bosu ball y ligeramente menor respecto respecto a los bloques foam (Drinkwater y cols., 2007). Otra investigación examinó la potencia ${ }_{\text {media }}$ en la fase concéntrica al realizar sentadillas al 70\% de la 1 RM (Zemková y cols., 2012). Los resultados mostraban menor potencia ${ }_{\text {media }}$, al realizar sentadilla encima del bosu ball. La misma tendencia fue observada ante diferentes cargas cuando se realizaban sentadillas con y sin contramovimiento (Zemková y cols., 2013), siendo la potencia ${ }_{\text {media }}$ menor en CI. Así, a pesar de la inestabilidad local generada por el Pielaster a nivel del tobillo (módulo de la aceleración $=3.94 \pm 1.27 \mathrm{~m} \cdot \mathrm{s}^{-2}$ ), realizar sentadillas con VP encima de Pielasters parece no afectar a la estabilidad global debido probablemente a la menor altura del centro de masas respecto a realizar sentadillas con barra encima de los hombros, y a la fijación del sujeto a la VP mediante la cuerda que ejerce una fuerza contra el suelo que podría facilitar la estabilidad del participante. Estas consideraciones podrían explicar la capacidad de producir velocidad $_{\text {pico }}$ de forma similar en ambas condiciones.

Las investigaciones que han comparado los efectos de dos programas de entrenamiento de fuerza en CE y CI en jóvenes previamente desentrenados indican que a pesar de utilizar cargas más ligeras, los efectos del programa de entrenamiento en CI mostraron una aumento de la fuerza (Marinkovic, Bratić, Ignjatović, y Radovanović, 2012; Sparkes y Behm, 2010) y del equilibrio (Sparkes y Behm, 2010) similar a la del programa realizado en $\mathrm{CE}$ que utilizaba cargas más pesadas. Por lo tanto,

La sentadilla realizada encima de Pielasters era el único ejercicio que incluía entrenamiento de las extremidades inferiores en CI. Por tanto, podríamos atribuir en gran parte la mejora de la velocidad ${ }_{\text {pico }}$ alcanzada en CI, al entrenamiento reiterado de esa tarea. El ratio velocidad ${ }_{\text {pico }}$ en $\mathrm{CE} /$ velocidaden CI fue $1,03(3,4 \%)$ en la primera sesión convirtiéndose en $0,98(-2,1 \%)$ en la última de las sesiones evaluadas. De esta forma, observamos una ligera tendencia a aumentar más la velocidad pico $_{\text {en }}$ CI que en CE. Esto podría estar en parte justificado por adaptaciones neuromusculares específicas al estímulo de entrenamiento (Cormie, McGuigan, y Newton, 2010), provocando mayor eficacia motora y optimización del rendimiento muscular. Además, realizar sentadillas encima de Pielasters podría incidir positivamente en la prevención de lesiones, especialmente esguinces de tobillos, debido al componente de inestabilidad y propioceptivo provocado por los Pielasters en esta articulación.

Las adaptaciones en la AM que tuvieron lugar durante la temporada podrían haber sido en parte resultado de adaptaciones a los mesociclos de entrenamiento de fuerza, donde el énfasis recaía principalmente en el entrenamiento con cargas medias y altas para el desarrollo de la hipertrofia muscular y de la potencia. Así, el grosor muscular en un grupo control de sujetos no entrenados era 2,32 $\pm 0,22$ (Abe, Kumagai, y Brechue, 2000), un 15,6\% inferior respecto al presente estudio. El ángulo de peneación $(19,5 \pm 3,6$ o $)$ y la longitud de los fascículos $(7,13 \pm 1,18 \mathrm{~cm})$ del grupo control (Abe y cols., 2000 ) eran un $3 \%$ y un $11 \%$ respectivamente mayores en los jugadores de baloncesto. Estas diferencias podrían deberse en cierta medida al entrenamiento de fuerza realizado por los jugadores de baloncesto frente a la inactividad del grupo control. Por otro lado, el grosor muscular del vasto lateral en sprinters de $100 \mathrm{~m}$ lisos era 2,75 $\pm 0,30 \mathrm{~cm}$ (Kumagai y cols., 2000) siendo igual al de los participantes en el presente estudio. El ángulo de peneación era 19,0 \pm 3,2 o un 5,6\% inferior, y la longitud de los fascículos $8,63 \pm 1,42 \mathrm{~cm}$, un $7,7 \%$ superior respecto a los resultados de los participantes del presente estudio. El incremento del 8,7 \% en el ángulo de peneación hallado en nuestro estudio era inferior al 36\% (Aagaard y cols., 2001) y al 28-35\% (Reeves y cols., 2004) obtenido en el vasto lateral después de realizar un programa 
de fuerza durante 14 semanas. Probablemente, estas diferencias sean debidas a que los sujetos no presentaban experiencia previa en el entrenamiento de fuerza (Aagaard y cols., 2001) y a que eran personas mayores (Reeves y cols., 2004) mientras que los jugadores de baloncesto presentaban experiencia en este tipo de entrenamiento y realizaban, además, el entrenamiento técnico-táctico. Aunque se desconoce con exactitud la influencia del crecimiento biológico de los jugadores en las adaptaciones producidas en este estudio, creemos que ésta no debería ser especialmente importante, ya que las pruebas distaban tan solo 3 meses.

Pocos estudios han abordado los cambios producidos en la AM del vasto lateral durante una temporada realizando el entrenamiento técnico-táctico de su deporte y de fuerza de forma concurrente (Blazevich y cols., 2003; Jajtner y cols., 2013; Nimphius y cols., 2012). Los resultados mostraron una reducción del 2,6 y del 6,8\% (Jajtner y cols., 2013) pero un incremento del 3,5 y del 4,2\% (Nimphius y cols., 2012) en el grosor muscular y en el ángulo de peneación respectivamente. Además, Nimphius y cols. (2012) hallaron un aumento del $9,5 \%$ en la longitud de los fascículos. Cabe destacar el $45 \%$ de diferencia entre el grosor muscular hallado en los estudios anteriores $(1,46 \pm 0,21$ y $2,70 \pm 0,38 \mathrm{~cm})$ situando a las jugadoras de softball al nivel de los sprinters de $100 \mathrm{~m}$ lisos citados anteriormente y de los jugadores de baloncesto de este estudio.

\section{Aplicaciones prácticas}

La sentadilla forma parte de los programas de entrenamiento para deportes que requieren altos niveles de fuerza y de po- tencia. El entrenamiento de fuerza realizando sentadillas con VP permitió alcanzar niveles de velocidad ${ }_{\text {pico }}$ muy similares en CE y CI. La velocidad ${ }_{\text {pico }}$ aumentó a lo largo de la temporada de forma similar en ambas condiciones.

De este modo, los hallazgos de este estudio indican que la realización de sentadillas utilizando el dispositivo de inercia rotacional VP con Pielasters podría provocar un mayor desafío neuromuscular en jóvenes jugadores de baloncesto de alto nivel especialmente en la articulación del tobillo sin disminuir la velocidad de ejecución, consiguiendo así una mayor especificidad e incidencia en la prevención de esquinces de tobillo. Por otra parte, este dispositivo proporciona una alternativa a realizar el ejercicio de sentadilla tradicional con peso libre y podría ser incluido en la periodización de programas de entrenamiento de fuerza.

Por otro lado, la AM podría ser incluida como una forma de control y valoración de las adaptaciones musculares producidas con el entrenamiento complementando las valoraciones de fuerza y potencia.

Agradecimientos.- Los autores agradecen al responsable de los servicios médicos del FCBarcelona, el doctor Ramon Canal por hacer posible esta investigación, así como a la doctora Gemma Hernández por realizar las ecografías. Además, agradecemos al Área de Rendimiento del FCBarcelona, concretamente al Sr. Joan Ramon Tarragó por las facilidades mostradas durante todo el estudio. A todos los jugadores del equipo junior de baloncesto su participación y compromiso. Este estudio ha sido realizado con el apoyo del Instituto Nacional de Educación Física de Catalunya (INEFC) de la Generalitat de Catalunya.

\section{Referencias}

1. Aagaard, P., Andersen, J.L., Dyhre-Poulsen, P., Leffers, A.-M., Wagner, A., Magnusson, S.P., . . Simonsen, E.B. (2001). A mechanism for increased contractile strength of human pennate muscle in response to strength training: changes in muscle architecture. The Journal of Physiology, 534(2), 613-623. doi: 10.1111/j.1469-7793.2001.t01-1-00613.x

2. Abe, T., Brechue, W.F., Fujita, S., y Brown, J.B. (1998). Gender differences in FFM accumulation and architectural characteristics of muscle. Medicine \& Science in Sports \& Exercise, 30(7), 1066-1070.

3. Abe, T., Kumagai, K., y Brechue, W.F. (2000). Fascicle length of leg muscles is greater in sprinters than distance runners. Medicine \& Science in Sports \& Exercise, 32(6), 1125-1129.

4. Albert, M.S., Hillegass, E., y Spiegel, P. (1994). Muscle torque changes caused by inertial exercise training. J Orthop Sports Phys Ther, 20(5), 254-261. doi: 10.2519/jospt.1994.20.5.254

5. Alegre, L.M., Jiménez, F., Gonzalo-Orden, J.M., Martín-Acero, R., y Aguado, X. (2006). Effects of dynamic resistance training on fascicle lengthand isometric strength. J Sports Sci, 24(5), 501-508. doi: 10.1080/02640410500189322

6. Apostolidis, N., Nassis, G.P., Bolatoglou, T., y Geladas, N.D. (2004). Physiological and technical characteristics of elite young basketball players. J Sports Med Phys Fitness, 44(2), 157-163.

7. Behm, D., y Colado, J.C. (2012). The effectiveness of resistance trai- ning using unstable surfaces and devices for rehabilitation. Int J Sports Phys Ther, 7(2), 226-241.

8. Behm, D.G., Anderson, K., y Curnew, R.S. (2002). Muscle force and activation under stable and unstable conditions. Journal of Strength and Conditioning Research, 16(3), 416-422.

9. Behm, D.G., y Anderson, K.G. (2006). The role of instability with resistance training. Journal of Strength and Conditioning Research, 20(3), 716-722. doi: 10.1519/r-18475.1

10. Behm, D.G., Leonard, A.M., Young, W.B., Bonsey, W.A., y MacKinnon, S.N. (2005). Trunk muscle electromyographic activity with unstable and unilateral exercises. Journal of Strength and Conditioning Research, 19(1), 193-201. doi: 10.1519/1533-4287(2005)19<193:tmeaw $\mathrm{u}>2.0 . \mathrm{co} ; 2$

11. Ben Abdelkrim, N., Castagna, C., Jabri, I., Battikh, T., El Fazaa, S., y Ati, J.E. (2010). Activity Profile and Physiological Requirements of Junior Elite Basketball Players in Relation to Aerobic-Anaerobic Fitness. The Journal of Strength \& Conditioning Research, 24(9), 2330-2342 2310.1519/JSC.2330b2013e3181e2381c2331.

12. Ben Abdelkrim, N., El Fazaa, S., y El Ati, J. (2007). Time-motion analysis and physiological data of elite under-19-year-old basketball players during competition. Br J Sports Med, 41(2), 69-75; discussion 75. doi: $10.1136 /$ bjsm.2006.032318 
13. Berg, H.E., y Tesch, A. (1994). A gravity-independent ergometer to be used for resistance training in space. Aviat Space Environ Med, 65(8), 752-756.

14. Berg, H.E., y Tesch, P.A. (1998). Force and power characteristics of a resistive exercise device for use in space. Acta Astronaut, 42(1-8), 219230 .

15. Blazevich, A.J., Cannavan, D., Coleman, D.R., y Horne, S. (2007). Influence of concentric and eccentric resistance training on architectural adaptation in human quadriceps muscles (Vol. 103).

16. Blazevich, A.J., Gill, N.D., Bronks, R., y Newton, R.U. (2003). Training-Specific Muscle Architecture Adaptation after 5-wk Training in Athletes. Medicine \& Science in Sports \& Exercise, 35(12), 2013-2022.

17. Blazevich, A.J., Gill, N.D., Deans, N., y Zhou, S. (2007). Lack of human muscle architectural adaptation after short-term strength training. Muscle \& Nerve, 35(1), 78-86. doi: 10.1002/mus.20666

18. Blazevich, A.J., y Giorgi, A. (2001). Effect of testosterone administration and weight training on muscle architecture. Medicine \& Science in Sports \& Exercise, 33(10), 1688-1693.

19. Caruso, J.F., Hernandez, D.A., Porter, A., Schweikert, T., Saito, K., Cho, M., . . . Nelson, N.M. (2006). Integrated Electromyography and Performance Outcomes to Inertial Resistance Exercise. The Journal of Strength \& Conditioning Research, 20(1), 151-156.

20. Comfort, P., Bullock, N., y Pearson, S.J. (2012). A Comparison of Maximal Squat Strength and 5-, 10-, and 20-Meter Sprint Times, in Athletes and Recreationally Trained Men. The Journal of Strength \& Conditioning Research, 26(4), 937-940 910.1519/ JSC.1510b1013e31822e35889.

21. Cormie, P., McGuigan, M.R., y Newton, R.U. (2010). Adaptations in Athletic Performance after Ballistic Power versus Strength Training. Medicine \& Science in Sports \& Exercise, 42(8), 1582-1598 1510.1249/ MSS.1580b1013e3181d2013a.

22. Chandler, T.J., Stone, Micheal H. (1992). The Squat Exercise in Athletic Conditioning: A Position Statement and Review of the Literature: Lincoln, NE: National Strength \& Concitioning Association (U.S.).

23. Drinkwater, E.J., Pritchett, E.J., y Behm, D.G. (2007). Effect of instability and resistance on unintentional squat-lifting kinetics. Int J Sports Physiol Perform, 2(4), 400-413.

24. Ehlenz, H., Grosser, M., y Zimmermann, E. (1990). Entrenamiento de la fuerza. Barcelona: Martínez Roca.

25. Hedrick, A. (1993). Strength and power training for basketball. National Strength and Conditioning Association Journal, 15(4), 31-35.

26. Hoffman, J.R., y Maresch, C.M. (2000). Physiology testing of basketball players. In W. E. Garrenty y D. T. Kirkendall (Eds.), Exercise and sport science (pp. 733-744). Philadelphia: Lippicott Williams y Wilkins.

27. Hoffman, J.R., Tenenbaum, G., Maresh, C.M., y Kraemer, W.J. (1996). Relationship Between Athletic Performance Tests and Playing Time in Elite College Basketball Players. The Journal of Strength \& Conditioning Research, 10(2), 67-71.

28. Jajtner, A.R., Hoffman, J.R., Scanlon, T.C., Wells, A.J., Townsend, J.R., Beyer, K.S., . . S Stout, J.R. (2013). Performance and Muscle Architecture Comparisons Between Starters and Nonstarters in National Collegiate Athletic Association Division I Women's Soccer. The Journal of Strength \& Conditioning Research, 27(9), 2355-2365 2310.1519/ JSC.2350b2013e31829bd31827c31825.

29. Kawakami, Y., Abe, T., y Fukunaga, T. (1993). Muscle-fiber pennation angles are greater in hypertrophied than in normal muscles (Vol. 74).

30. Kawakami, Y., Abe, T., Kuno, S.Y., y Fukunaga, T. (1995). Traininginduced changes in muscle architecture and specific tension. Eur $J$ Appl Physiol Occup Physiol, 72(1-2), 37-43.

31. Kearns, C.F., Isokawa, M., y Abe, T. (2001). Architectural characteristics of dominant leg muscles in junior soccer players. Eur J Appl Physiol, $85(3-4), 240-243$.
32. Klinzing, J.E. (1991). Training for improved jumping ability of basketball players. Strength and Conditioning Journal, 13(3), 27-33.

33. Kubo, K., Kanehisa, H., Azuma, K., Ishizu, M., Kuno, S.-y., Okada, M., y Fukunaga, T. (2003). Muscle Architectural Characteristics in Women Aged 20-79 Years. Medicine \& Science in Sports \& Exercise, 35(1), 39-44.

34. Kumagai, K., Abe, T., Brechue, W.F., Ryushi, T., Takano, S., y Mizuno, M. (2000). Sprint performance is related to muscle fascicle length in male 100-m sprinters (Vol. 88).

35. Marinkovic, M., Bratić, M., Ignjatović, A., y Radovanović, D. (2012) Effects of 8-Week instability resistance training on maximal strength in inexperienced young individuals. Servian Journal of Sports Sciences, 6(1), 17-21.

36. McBride, J.M., Blow, D., Kirby, T.J., Haines, T.L., Dayne, A.M., y Triplett, N.T. (2009). Relationship Between Maximal Squat Strength and Five, Ten, and Forty Yard Sprint Times. The Journal of Strength \& Conditioning Research, 23(6), 1633-1636 1610.1519/JSC.1630b1013e3 181b1632b1638aa.

37. McGuigan, M.R., Wright, G.A., y Fleck, S.J. (2012). Strength training for athletes: does it really help sports performance? Int J Sports Physiol Perform, 7(1), 2-5.

38. McInnes, S.E., Carlson, J.S., Jones, C.J., y McKenna, M.J. (1995). The physiological load imposed on basketball players during competition. J Sports Sci, 13(5), 387-397. doi: 10.1080/02640419508732254

39. Naczk, M., Naczk, A., Brzenczek-Owczarzak, W., Arlet, J., y Adach, Z. (2013). Impact of inertial training on strength and power performance in young active men. J Strength Cond Res. doi: 10.1519/ JSC.0b013e3182a993c2

40. Naczk, M., Naczk, A., Brzenczek-Owczarzak, W., Arlet, J., y Adach, Z (2013). Impact of inertial training on strength and power performance in young active men. The Journal of Strength \& Conditioning Research, Publish Ahead of Print, 10.1519/JSC.1510b1013e3182a1993c1512.

41. Nimphius, S., McGuigan, M.R., y Newton, R.U. (2012). Changes in Muscle Architecture and Performance During a Competitive Season in Female Softball Players. The Journal of Strength \& Conditioning Research, 26(10), 2655-2666 2610.1519/JSC.2650b2013e318269f318281e.

42. Norrbrand, L., Fluckey, J., Pozzo, M., y Tesch, P. (2008). Resistance training using eccentric overload induces early adaptations in skeletal muscle size. Eur J Appl Physiol, 102(3), 271-281. doi: 10.1007/s00421007-0583-8

43. Norrbrand, L., Pozzo, M., y Tesch, P. (2010). Flywheel resistance training calls for greater eccentric muscle activation than weight training. Eur J Appl Physiol, 110(5), 997-1005. doi: 10.1007/s00421-010-1575-7

44. Onambélé, G.L., Maganaris, C.N., Mian, O.S., Tam, E., Rejc, E., McEwan, I.M., y Narici, M.V. (2008). Neuromuscular and balance responses to flywheel inertial versus weight training in older persons. Journal of biomechanics, 41(15), 3133-3138.

45. Oshita, K., y Yano, S. (2010). Relationship between force fluctuation in the plantar flexor and sustainable time for single-leg standing. $J$ Physiol Anthropol, 29(3), 89-93.

46. Reeves, N.D., Narici, M.V., y Maganaris, C.N. (2004). In vivo human muscle structure and function: adaptations to resistance training in old age. Experimental Physiology, 89(6), 675-689. doi: 10.1113/expphysiol.2004.027797

47. Rutherford, O.M., y Jones, D.A. (1992). Measurement of fibre pennation using ultrasound in the human quadriceps in vivo. Eur J Appl Physiol Occup Physiol, 65(5), 433-437.

48. Saeterbakken, A.H., y Fimland, M.S. (2013). Muscle force output and electromyographic activity in squats with various unstable surfaces. J Strength Cond Res, 27(1), 130-136. doi: 10.1519/ JSC.0b013e3182541d43

49. Sallet, P., Perrier, D., Ferret, J.M., Vitelli, V., y Baverel, G. (2005). Physiological differences in professional basketball players as a function 
of playing position and level of play. J Sports Med Phys Fitness, 45(3), 291-294.

50. Seynnes, O.R., de Boer, M., y Narici, M.V. (2007). Early skeletal muscle hypertrophy and architectural changes in response to high-intensity resistance training (Vol. 102).

51. Simenz, C.J., Dugan, C.A., y Ebben, W.P. (2005). Strength and conditioning practices of National Basketball Association strength and conditioning coaches. J Strength Cond Res, 19(3), 495-504. doi: 10.1519/15264.1

52. Sparkes, R., y Behm, D.G. (2010). Training Adaptations Associated With an 8-Week Instability Resistance Training Program With Recreationally Active Individuals. The Journal of Strength \& Conditioning Research, 24(7), 1931-1941 1910.1519/JSC.1930b1013e3181df1937 fe1934.

53. Tesch, P.A., Ekberg, A., Lindquist, D.M., y Trieschmann, J.T. (2004).
Muscle hypertrophy following 5-week resistance training using a non-gravity-dependent exercise system. Acta Physiologica Scandinavica, 180(1), 89-98. doi: 10.1046/j.0001-6772.2003.01225.x

54. Wickiewicz, T.L., Roy, R.R., Powell, P.L., y Edgerton, V.R. (1983). Muscle architecture of the human lower limb. Clin Orthop Relat $\operatorname{Res}(179), 275-283$.

55. Zemková, E., Jelen, M., Kovacikova, Z., Olle, G., Vilman, T., y Hamar, D. (2013). Weight Lifted and Countermovement Potentiation of Power in Concentric Phase of Unstable and Traditional Resistance Exercise. J Appl Biomech.

56. Zemková, E., Jelen, M., Kováciková, Z., Ollé, G., Vilman, T., y Hamar, D. (2012). Power Outputs in the Concentric Phase of Resistance Exercises Performed in the Interval Mode on Stable and Unstable Surfaces. The Journal of Strength \& Conditioning Research, 26(12), 3230$32363210.1519 /$ JSC.3230b3013e31824bc31197. 